\title{
Grapheme-color synesthesia can enhance immediate memory without disrupting the encoding of relational cues
}

\author{
Bradley S. Gibson • Gabriel A. Radvansky • \\ Ann C. Johnson • M. Windy McNerney
}

Published online: 27 July 2012

(C) Psychonomic Society, Inc. 2012

\begin{abstract}
Previous evidence has suggested that graphemecolor synesthesia can enhance memory for words, but little is known about how these photisms cue retrieval. Often, the encoding of specific features of individual words can disrupt the encoding of ordered relations between words, resulting in an overall decrease in recall accuracy. Here we show that the photisms arising from grapheme-color synesthesia do not function like these item-specific cues. The influences of high and low word frequency on the encoding of ordered relations and the accuracy of immediate free recall were compared across a group of 10 synesthetes and 48 nonsynesthetes. The main findings of Experiment 1 showed that the experience of synesthesia had no adverse effect on the encoding of ordered relations (as measured by input-output correspondence); furthermore, it enhanced recall accuracy in a strictly additive fashion across the two word frequency conditions. Experiment 2 corroborated these findings by showing that the synesthetes only outperformed the nonsynesthetes when the materials involved words and letters, not when they involved digits and spatial locations. Altogether, the present findings suggest that synesthesia can boost immediate memory performance without disrupting the encoding of ordered relations.
\end{abstract}

Keywords Retrieval cues and memory · Working memory · Synesthesia

Synesthesia is a condition in which a person reports inappropriate and involuntary sensory experiences in addition to the standard ones (see Grossenbacher \& Lovelace, 2001;

B. S. Gibson $(\bowtie) \cdot$ G. A. Radvansky • A. C. Johnson •

M. W. McNerney

Department of Psychology, University of Notre Dame,

118 Haggar Hall,

Notre Dame, IN 46556, USA

e-mail: gibson.16@nd.edu
Hochel \& Milán, 2008; Hubbard \& Ramachandran, 2005; Rich \& Mattingly, 2002, for reviews). Of particular concern here are those cases in which a person experiences colors (photisms) when reading words - a condition known as "grapheme-color synesthesia" (GCS). These letter-color associations are unique to each synesthete, although there are regularities across large numbers of synesthetes (Simner et al., 2005). GCS is also one of the more common types of synesthesia, occurring in $45 \%$ to $65 \%$ of synesthetes (Simner et al., 2006). While a number of studies have addressed how GCS affects perception and attention, relatively few studies have addressed how this condition affects memory. Accordingly, the present study examines how GCS affects cue-dependent retrieval from immediate memory.

Anecdotally, people with GCS tend to self-report that they have better memories for letters and words than most people (Luria, 1968; Tammet, 2009; Yaro \& Ward, 2007). Radvansky, Gibson, and McNerney (2011) attempted to verify these subjective reports by administering multiple memory tests to a group of $10 \mathrm{GC}$ synesthetes and 48 nonsynesthetes. They found that the GC synesthetes had superior memory for word lists as compared to the nonsynesthetes. On the basis of this finding, Radvansky et al. concluded that GCS can enhance memory capacity for word lists because the photisms that are elicited by the orthography of each word can be used as retrieval cues by those who experience them (see also Mills, Innis, Westendorf, Owsianiecki, \& McDonald, 2006; Smilek, Dixon, Cudahy, \& Merikle, 2002; Ward, 2008).

There has been long-standing interest in understanding the general principles that govern cue-dependent retrieval from immediate memory (Brown, Neath \& Chater, 2007; Nairne, 2002). For instance, Hunt and Einstein (1981) suggested that immediate free recall is governed by the interplay between at least two kinds of retrieval cues: those that are derived from the relations between items, and those that are derived from the specific features of individual items. Furthermore, these relational and item-specific cues are 
thought to serve different functional roles during recall. Relational cues may provide general structure to guide the retrieval of target candidates, whereas item-specific cues may provide information that allows current list candidates to be discriminated from other candidates not on the current list (Hunt \& Einstein, 1981; McDaniel \& Bugg, 2008).

Within this context, the photisms that arise from GCS may serve as item-specific cues because they are elicited by the individual letters and words (Simner, 2007) and do not convey relational information across words. Thus, synesthetes may have access to an additional source of itemspecific information relative to nonsynesthetes. But, do these synesthetic cues follow the same principles as those arising from other item-specific cues?

One way to address this question is to consider the encoding dynamics of relational and item-specific features when memory list composition is manipulated. On the one hand, there is evidence that the encoding of relational and item-specific features can have independent effects on recall in nonsynesthetes, at least under some conditions. For instance, Hunt and Mitchell (1982) provided evidence that the encoding of semantic relations and orthographic distinctiveness can have additive effects on recall. However, Radvansky et al. (2011) concluded that the encoding of synesthetic photisms appeared to decrease emphasis on the encoding of semantic relations, as evidenced by smaller or absent von Restorff effects when a list singleton was identified by a unique semantic meaning and by smaller Deese/Roediger-McDermott false memory effects. In other words, the encoding of synesthetic item-specific cues appeared to disrupt the encoding of semantic relational cues.

On the other hand, the effects of synesthesia on cuedependent retrieval may not be that unusual, because there is also evidence that relational and item-specific retrieval cues can trade off in nonsynesthetes as well. For instance, consider the encoding dynamics of serial order and itemspecific features when pure lists of high- and low-frequency words are presented in the context of immediate free recall. According to the item-order account (McDaniel \& Bugg, 2008), the ordered relations between list items tend to be encoded at the expense of the specific features of individual list items for pure lists of high-frequency words, whereas these features of list items tend to be encoded at the expense of the ordered relations for pure lists of low-frequency words. This trade-off is thought to occur because highfrequency words have relatively ordinary individual features that do not attract (or demand) attention, whereas lowfrequency words have relatively unordinary individual features that do.

Tests of the item-order account have primarily focused on two outcome measures: input-output correspondence and recall accuracy. Input-output correspondence is an implicit measure of the extent to which the relative serial order of the list items is reflected in the recall response (Asch \& Ebenholtz, 1962; Nairne, Riegler, \& Serra, 1991), and recall accuracy is an explicit measure of the proportion of items correctly recalled. According to the item-order account, input-output correspondence should be greater for pure high-frequency lists than for pure lowfrequency lists, because serial order is encoded to a greater extent in high-frequency lists. Moreover, recall accuracy should be greater for pure high-frequency lists than for pure low-frequency lists, because serial order cues are more potent than item-specific cues. Consistent with this, people tend to achieve greater input-output correspondence and recall accuracy in free recall for pure high-frequency lists than for pure low-frequency lists (McDaniel \& Bugg, 2008).

In the present study, we examined whether the itemspecific cues arising from GCS function like those arising from low-frequency words by examining whether group differences in the experience of synesthesia affect the dynamics of item-order encoding for pure high- and lowfrequency word lists. More specifically, synesthetes may be more likely to engage in item-specific encoding than are nonsynesthetes, regardless of list type, because the synesthetic experience draws attention to the shallow, surface form features of each word (Radvansky et al., 2011). In considering this prediction, it is important to note that word frequency is not expected to otherwise interact with the quality of the synesthetic experience (Ward, Simner, \& Auyeung, 2005).

If these synesthetic item-specific cues function like lowfrequency words, then GCS should alter the dynamics of item-order encoding, because the encoding of these photisms may disrupt serial-order encoding during list presentation, which may in turn cause a decrease in input-output correspondence (McDaniel \& Bugg, 2008), especially for pure lists of high-frequency words. The top panel in Fig. 1 shows this prediction, with input-output correspondence being greater when high-frequency pure lists are shown to nonsynesthetes relative to when low-frequency pure lists are shown. However, this advantage is reduced (or eliminated) when these lists are shown to synesthetes.

Furthermore, if there is decreased input-output correspondence for the synesthetes, then there should also be decreased recall accuracy, especially for high-frequency pure lists. However, a decrease in recall accuracy stemming from the disrupted serial-order encoding may be masked or offset by increases in recall accuracy because of additional item-specific encoding in both wordfrequency conditions. The bottom panel of Fig. 1 shows the predicted pattern of recall if GCS disrupts serialorder encoding. Recall accuracy should be greater when high-frequency pure lists are shown to nonsynesthetes relative to when low-frequency pure lists are shown, but 


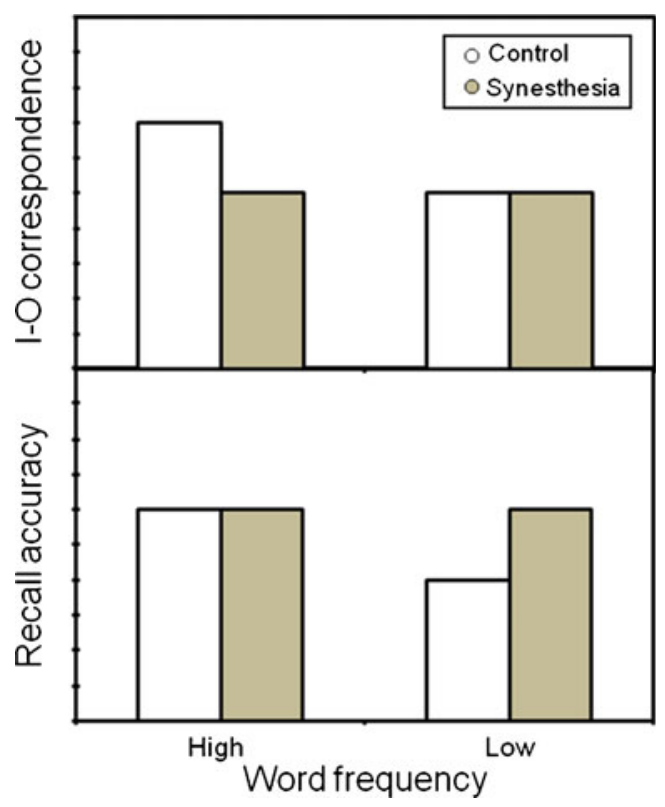

Fig. 1 Predicted effects of group and word frequency if synesthesia disrupts the encoding of ordered relations. The top panel shows inputoutput correspondence, and the bottom panel shows recall accuracy

this difference should be reduced (or eliminated) when these lists are shown to synesthetes. More importantly, when recall accuracy is compared across groups, recall accuracy should be greater for synesthetes than for nonsynesthetes when low-frequency pure lists are shown, but not when high-frequency pure lists are shown.

In contrast, another possibility is that GCS does not alter the dynamics of item-order encoding, because these cues do not function like low-frequency words. If so, then inputoutput correspondence should be greater when highfrequency pure lists are shown relative to when lowfrequency pure lists are shown, and the same difference should be observed across both groups (see the top panel of Fig. 2). Likewise, recall accuracy should also be greater when high-frequency pure lists are shown relative to when low-frequency pure lists are shown, and the same difference should once again be observed across both groups (see bottom panel of Fig. 2). Most importantly, the synesthetic item-specific cues may serve as retrieval cues in their own right, in which case recall accuracy should be higher overall for the synesthetes than for the nonsynesthetes (see the bottom panel of Fig. 2).

In summary, pure lists of high- and low-frequency words were presented to synesthetes and nonsynesthetes in the present study. By comparing the word frequency influences on input-output correspondence and recall accuracy across the two groups, the present study can determine whether the item-specific cues arising from GCS conform to the same principles as those arising from low-frequency words.

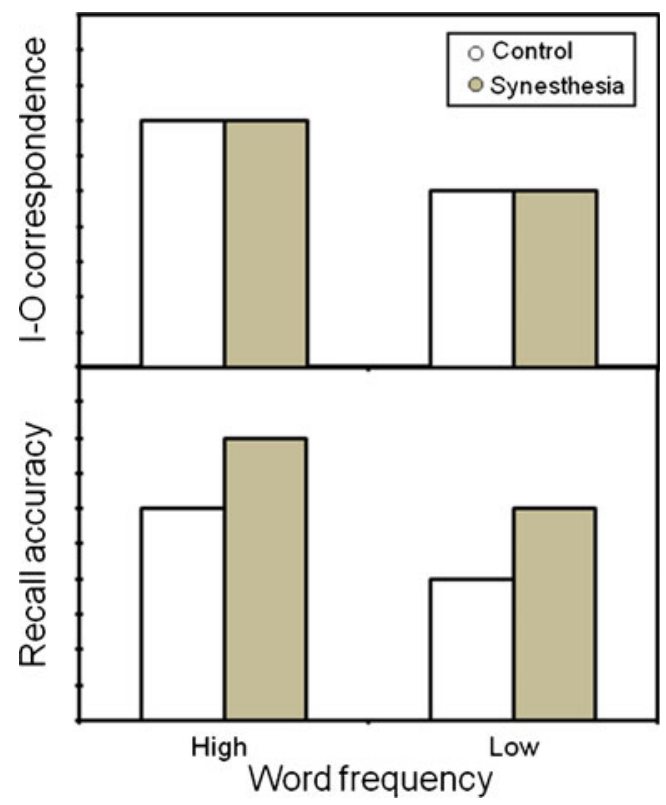

Fig. 2 Predicted effects of group and word frequency if synesthesia does not disrupt the encoding of ordered relations. The top panel shows input-output correspondence, and the bottom panel shows recall accuracy

\section{Experiment 1}

Method

Participants A total of 58 undergraduates from the University of Notre Dame participated in this experiment. Ten of these participants were synesthetes (eight female) who reported experiencing colors as they read different letters (see Radvansky et al., 2011, for a fuller description of how the synesthetic experience reported by these participants was tested and confirmed). The remaining 48 undergraduates did not report any synesthetic experiences. All of the synesthetes reported that the photisms were experienced in their minds as opposed to in the world (Dixon, Smilek, \& Merikle, 2004).

On the basis of our understanding of the word frequency effect (McDaniel \& Bugg, 2008) and the synesthete effect (Radvansky et al., 2011), we expected the effect sizes $\left(\eta_{\mathrm{p}}{ }^{2}\right)$ associated with the two main effects depicted in Fig. 2 or the interaction depicted in Fig. 1 to be very large (greater than 0.16 ). On the basis of this assumption, the power was at least .80 to detect these effects with the sample sizes that were used.

Immediate free recall task We created 24 lists, each containing 12 words. The words were all four- to eightletter verbs $(M=5.4)$ that contained one to three syllables each $(M=1.6)$. Twelve of the word lists contained only lowfrequency words, which occurred 1 time per million in the Francis and Kučera (1982) word norms, and the other 12 of the word lists contained only high-frequency words, occurring 
176 to 3,941 per million $(M=455)$ in the Francis and Kučera norms. All of the words within a list were semantically unrelated, and the presentation of words within each list was randomized. At the end of each list, people were prompted to recall as many items as possible, regardless of order. During recall, people typed their responses into the computer. The computer allowed only the current response to be viewed.

Data analyses The order and number of correct and incorrect recall responses were recorded for each person. A response was scored as correct only if it matched one of the list items verbatim. Two outcome measures were derived from the immediate free recall task: input-output correspondence and recall accuracy. Input-output correspondence was based on the technique developed by Asch and Ebenholtz (1962; see also Nairne et al., 1991). This measure provides a relative index of correct input-output correspondence. For example, suppose that a participant recalled, in order, Items 2, 3, 7, 6, and 4 from the 12-item list. By evaluating the relative order of each successive pair of recall responses, input-output correspondence is a proportion equal to the number of these pairs that preserve the relative order of the input $(2-3,3-7)$ divided by the total number of pairs $(2-3,3-7,7-6$, and $6-4)$. Thus, the input-output correspondence equals .50 on this example trial. Note that this measure takes into account correct adjacent (2-3) and remote (3-7) pairs, as well as the total number of pairs recalled. In contrast, recall accuracy is a proportion equal to the number of items correctly recalled (here, five) divided by the total number of list items (12). Thus, recall accuracy equals .42 on this example trial.

\section{Results and discussion}

The results for the two outcome measures are shown in Fig. 3 as a function of word frequency and group. A twoway mixed ANOVA was conducted on the two outcome measures. Word Frequency (high vs. low) was the sole within-subjects factor, and Group (synesthete vs. nonsynesthete) was the sole between-subjects factor in both analyses.

With respect to input-output correspondence, the results suggested that GCS did not interrupt serial-order encoding. As expected, input-output correspondence was greater for the high-frequency pure lists $(M=.68, S E=.02)$ than for the low-frequency pure lists $(M=.54, S E=.03), F(1,56)=$ $45.08, p<.0001, \eta_{\mathrm{p}}{ }^{2}=.446$. In addition, the estimate of input-output correspondence was significantly greater than chance (.50) for the high-frequency pure lists, $t(57)=12.25$, $p<.0001$, but not for the low-frequency pure lists, $t(57)=$ $1.56, p>.10$. This finding supports the idea that people used serial order to guide recall for high-frequency words but not for low-frequency words. More importantly, as can be seen in the top panel of Fig. 3, the same pattern was observed for both the synesthete and nonsynesthete groups. Consequently,

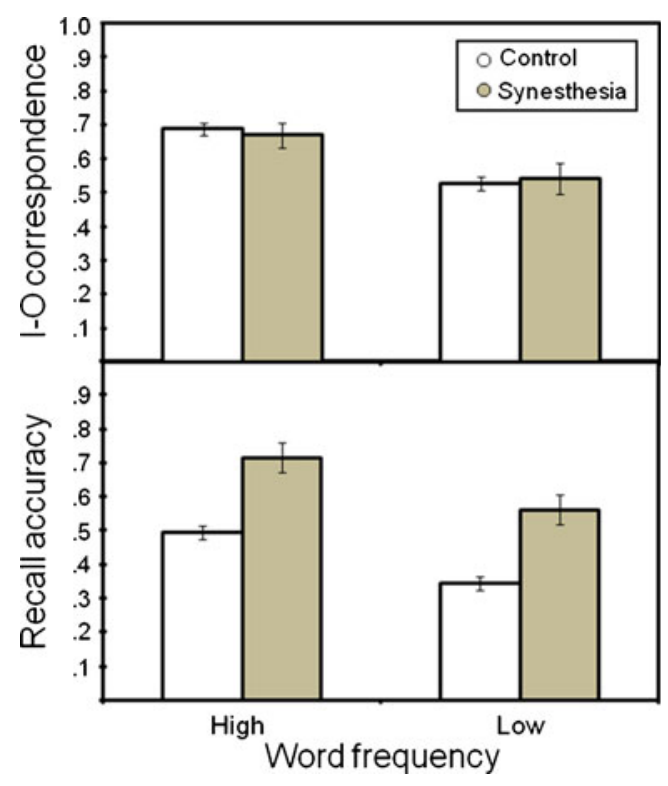

Fig. 3 Results of Experiment 1, depicted as a function of word frequency in each of the two groups. The top panel shows input-output correspondence, and the bottom panel shows recall accuracy. The error bars reflect standard errors

neither the main effect of group nor the interaction approached significance for input-output correspondence (both $F_{\mathrm{s}}<1, \eta_{\mathrm{p}}^{2}=.000$ and .010 , respectively).

The analysis of input-output correspondence suggests that the photisms arising from GCS do not disrupt the typical encoding of serial order in pure lists of highand low-frequency words. Therefore, one would also expect to observe the same effect of word frequency on recall accuracy across both groups. Consistent with this expectation, recall accuracy was greater for the highfrequency pure lists $(M=.60, S E=.03)$ than for the lowfrequency pure lists $(M=.45, S E=.02), F(1,56)=157.55, p<$ $.0001, \eta_{\mathrm{p}}{ }^{2}=.738$. In addition, recall accuracy was also greater for the synesthetes $(M=.64, S E=.04)$ than for the nonsynesthetes $(M=.42, S E=.02), F(1,56)=21.93, p<$ $.0001, \eta_{\mathrm{p}}{ }^{2}=.281$, suggesting that the photisms can serve as additional item-specific retrieval cues. Furthermore, as can be seen in the bottom panel of Fig. 3, the benefit of this additional item-specific retrieval cue was nearly identical for both highand low-frequency words, resulting in a nonsignificant interaction $\left(F<1, \eta_{\mathrm{p}}{ }^{2}=.001\right)$. However, before the memory advantage observed in the synesthete group could be clearly attributed to GCS, we had to show that the synesthetes did not have superior memory overall.

\section{Experiment 2}

Four simple span tasks were used. Two of the simple span tasks (letter span and word span) were expected to reveal 
group differences because the list items elicited photisms in the synesthetes. In contrast, two of the simple span tasks (digit span and location span) were not expected to reveal any group differences, because the list items did not elicit photisms in the synesthetes.

\section{Method}

Participants The participants were identical to those used in Experiment 1.

Simple span tasks Lists of 2-15 digits, letters, spatial locations, or words were presented on a computer screen. The digits and letters were randomly selected from the set of 10 or 26 possible items, respectively, and repetition was allowed within a set. The words were drawn from a pool of 385 three- to five-letter, single-syllable nouns that ranged in frequency from 50 to 4,393 per million (Francis \& Kučera, 1982). Within each list, the digits, letters, and words were presented sequentially for $1 \mathrm{~s}$ each. The spatial location task was modeled after that of Barton, Matthews, Farmer, and Belyavin (1995). In this task, squares appeared sequentially, for $2 \mathrm{~s}$ each, within a $6 \times 6$ grid.

Each span test started with a two-item list. At the end of each list of digits, letters, or words, participants were prompted to recall as many items as possible in the order that they had been learned. During recall, the participants typed their responses into the computer. The computer allowed only the current response to be viewed. Responses that included incorrect or misordered items were scored as errors. At the end of each list of spatial locations, participants were prompted to click on the locations that had been presented. Regardless of the task, five lists were shown at each span level, and the program did not advance to the next level unless at least one of the five lists at the current level had been recalled correctly. The task terminated when the participant was unable to achieve $100 \%$ accuracy on at least one list of a given length. Simple span scores were calculated following Turner and Engle's (1989) method, according to which the scores reflect the sum of the list lengths that were recalled with perfect accuracy.

Results and discussion

Simple span scores are reported in Table 1. Consistent with our expectations, the synesthetes achieved higher span scores than the nonsynesthetes on both the letter span task, $F(1,56)=9.08, p<.005$, and the word span task, $F(1,56)=$ $16.58, p<.001$. In contrast, the synesthetes did not achieve higher spans on either the digit span task, $F(1,56)<1$, or the location span task, $F(1,56)<1$. Hence, the synesthetes did not have superior memory overall.
Table 1 Total-correct scores as a function of group in each of the four simple span tasks used in Experiment 2

\begin{tabular}{lll}
\hline & Synesthetes & Controls \\
\hline Digit span & $129(12.6)$ & $119(6.7)$ \\
Letter span & $146(22.5)$ & $99(5.5)$ \\
Location span & $110(12.0)$ & $101(6.2)$ \\
Word span & $99(18.2)$ & $58(2.8)$ \\
\hline
\end{tabular}

Standard errors are shown in parentheses.

\section{General discussion}

In the present study, we investigated how differences in word frequency influence measures of input-out correspondence and recall accuracy across groups of grapheme-color synesthetes and nonsynesthetes. Our findings showed that the photisms arising from GCS can enhance the overall recall accuracy of unrelated word lists. But, more importantly, the present findings suggest that these photisms can enhance recall accuracy without incurring a trade-off between the encoding of item-specific features and serial order (see McDaniel \& Bugg, 2008, for a review). Whereas lowfrequency word lists decreased the encoding of the ordered relations between items relative to high-frequency word lists (as measured by input-output correspondence), the experience of GCS had no effect on the encoding of ordered relations between items, relative to nonsynesthetes. Consequently, high-frequency words were recalled more accurately than low-frequency words, regardless of group.

We acknowledge that this conclusion is based on two nonsignificant interactions, and that the present study was likely underpowered to detect the small $\left(\eta_{\mathrm{p}}{ }^{2}=.01\right)$ to very small $\left(\eta_{\mathrm{p}}{ }^{2}=.001\right)$ effect sizes that were actually observed post hoc (as opposed to the very large effect sizes that were predicted a priori in Fig. 1). However, although the analysis of input-output correspondence suggested a very slight trend in the direction predicted in Fig. 1, the analysis of recall accuracy suggested a very slight trend in the opposite direction. Thus, the only viable alternative hypothesis associated with these nonsignificant interactions does not correspond to any known theory. For this reason, we favor the null hypothesis associated with these interactions, depicted in Fig. 2.

Hence, the photisms that are encoded into memory and used during retrieval to enhance memory performance appear to operate in a different manner than the item-specific retrieval cues described by McDaniel and Bugg (2008). Like itemspecific cues, these photisms are based on the specific (orthographic) features of individual words. However, unlike the item-specific cues, the photisms do not appear to distract attention away from the encoding of serial order, perhaps because the photisms are an ordinary, and automatically 
occurring, aspect of the synesthetic experience. Indeed, Hunt and Mitchell (1982) proposed a similar mechanism to explain how nonsynesthetes may encode the orthographic distinctiveness of individual items without disrupting the encoding of semantic relations between items.

That said, it is puzzling that GCS did appear to disrupt the encoding of semantic relations in our previous study (Radvansky et al., 2011). However, closer examination of these findings suggests a less disparate conclusion: Namely, accuracy was indeed higher in the related condition $(M=.68$; see Exps. 3 and 4$)$ than in the unrelated condition $(M=.54$; see the control conditions from Exps. 1 and 2). In addition, accuracy was also consistently higher in the synesthete group $(M=.72)$ than in the nonsynesthete group $(M=.50)$. Importantly, the benefits of semantic relatedness were similar in the synesthete $(M=.15)$ and nonsynesthete $(M=.13)$ groups. So, considered in this way, these findings are consistent with the present findings, because they suggest that the retrieval benefits afforded by synesthesia are independent of the benefits afforded by semantic relations.

In conclusion, the present findings corroborate other findings suggesting that the features of the synesthetic experience are encoded into memory and used during retrieval to boost memory performance (Radvansky et al., 2011). More importantly, the present findings clarify and extend our prior findings by providing evidence that synestheticbased cues can boost memory performance without disrupting the encoding of relational cues. Instead, synestheticbased cues appear to influence retrieval with little or no cost to other forms of cue-dependent retrieval.

\section{References}

Asch, S. E., \& Ebenholtz, S. M. (1962). The process of free recall: Evidence for non-associate factors in acquisition and retention. Journal of Psychology: Interdisciplinary and Applied, 54, 3-31.

Barton, A., Matthews, B., Farmer, E., \& Belyavin, A. (1995). Revealing the basic properties of the visuospatial sketchpad: The use of complete spatial arrays. Acta Psychologica, 89, 197-216. doi:10.1016/0001-6918(95)98944-Y

Brown, G. D. A., Neath, I., \& Chater, N. (2007). A temporal ratio model of memory. Psychological Review, 114, 539-576. doi:10.1037/0033-295X.114.3.539

Dixon, M. J., Smilek, D., \& Merikle, P. M. (2004). Not all synaesthetes are created equal: Projector versus associator synaesthetes. Cognitive, Affective, \& Behavioral Neuroscience, 4, 335-343. doi:10.3758/CABN.4.3.335

Francis, W. N., \& Kučera, H. (1982). Frequency analysis of English usage: Lexicon and grammar. Boston, MA: Houghton Mifflin.

Grossenbacher, P. G., \& Lovelace, C. T. (2001). Mechanisms of synesthesia: Cognitive and physiological constraints. Trends in
Cognitive Sciences, 5, 36-41. doi:10.1016/S1364-6613(00) 01571-0

Hochel, M., \& Milán, E. G. (2008). Synaesthesia: The existing state of affairs. Cognitive Neuropsychology, 25, 93-117. doi:10.1080/ 02643290701822815

Hubbard, E. M., \& Ramachandran, V. S. (2005). Neurocognitive mechanisms of synesthesia. Neuron, 48, 509-520. doi:10.1016/ j.neuron.2005.10.012

Hunt, R. R., \& Einstein, G. O. (1981). Relational and item-specific information in memory. Journal of Verbal Learning \& Verbal Behavior, 20, 497-514.

Hunt, R. R., \& Mitchell, D. B. (1982). Independent effects of semantic and nonsemantic distinctiveness. Journal of Experimental Psychology: Learning, Memory, and Cognition, 8, 81-87. doi:10.1037/0278-7393.8.1.81

Luria, A. (1968). The mind of a mnemonist. New York, NY: Basic Books.

McDaniel, M. A., \& Bugg, J. M. (2008). Instability in memory phenomena: A common puzzle and a unifying explanation. Psychonomic Bulletin \& Review, 15, 237-255. doi:10.3758/ PBR.15.2.237

Mills, C. B., Innis, J., Westendorf, T., Owsianiecki, L., \& McDonald, A. (2006). Effect of a synesthete's photisms on name recall. Cortex, 42, 155-163.

Nairne, J. S. (2002). Remembering over the short-term: The case against the standard model. Annual Review of Psychology, 53, 53-81. doi:10.1146/annurev.psych.53.100901.135131

Nairne, J. S., Riegler, G. L., \& Serra, M. (1991). Dissociative effects of generation on item and order retention. Journal of Experimental Psychology: Learning, Memory, and Cognition, 17, 702-709. doi:10.1037/0278-7393.17.4.702

Radvansky, G. A., Gibson, B. S., \& McNerney, M. W. (2011). Synesthesia and memory: Color congruency, von Restorff, and false memory effects. Journal of Experimental Psychology: Learning, Memory, and Cognition, 37, 219-229. doi:10.1037/a0021329

Rich, A. N., \& Mattingly, J. B. (2002). Anomalous perception in synaesthesia: A cognitive neuroscience perspective. Nature Reviews Neuroscience, 3, 43-51. doi:10.1038/nrn702

Simner, J. (2007). Beyond perception: Synaesthesia as a psycholinguistic phenomenon. Trends in Cognitive Sciences, 11, 23-29. doi:10.1016/j.tics.2006.10.010

Simner, J., Mulvenna, C., Sagiv, N., Tsakanikos, E., Witherby, S. A., Fraser, C., et al. (2006). Synaesthesia: The prevalence of atypical cross-modal experiences. Perception, 35, 1024-1033. doi: $10.1068 /$ p5 569

Simner, J., Ward, J., Lanz, M., Jansari, A., Noonan, K., Glover, L., \& Oakley, D. A. (2005). Non-random associations of graphemes to colours in synaesthetic and non-synaesthetic populations. Cognitive Neuropsychology, 22, 1069-1085. doi:10.1080/ 02643290500200122

Smilek, D., Dixon, M. J., Cudahy, C., \& Merikle, P. M. (2002). Synesthetic color experiences influence memory. Psychological Science, 13, 548-552.

Tammet, D. (2009). Embracing the wide sky: A tour across the horizons of the mind. New York, NY: Free Press.

Turner, M. L., \& Engle, R. W. (1989). Is working memory capacity task dependent? Journal of Memory and Language, 28, 127-154.

Ward, J. (2008). The frog who croaked blue. New York, NY: Routledge.

Ward, J., Simner, J., \& Auyeung, V. (2005). A comparison of lexicalgustatory and grapheme-colour synaesthesia. Cognitive Neuropsychology, 22, 28-41. doi:10.1080/02643290442000022

Yaro, C., \& Ward, J. (2007). Searching for Shereshevskii: What is superior about the memory of synaesthetes? Quarterly Journal of Experimental Psychology, 60, 681-695. doi:10.1080/ 17470210600785208 